\title{
ESTUDO PARA REDUÇÃO DE PERMANÊNCIA DE VEÍCULOS APOIADO PELA SIMULAÇÃO EM COMPUTADOR*
}

\author{
Gustavo Nucci Franco ${ }^{1}$ \\ Marcellus Vinagre da Silva ${ }^{2}$ \\ Daniel Camargos Frade ${ }^{3}$ \\ Arthur Japiassu Marques Junior ${ }^{4}$ \\ Pedro Cesar Sinhoroto Machado ${ }^{5}$
}

\section{Resumo}

A indústria do aço passa por seu pior período no Brasil e as empresas vêm buscando soluções para minimizar custos e garantir as operações com menos recursos. Ponto importante nessa busca de ganhos potenciais, a Logística vem, assim, merecendo uma atenção especial. Este trabalho apresenta esforços da AM Juiz de Fora, em parceria com a Franco Arbeit Engenharia, para reduzir o tempo de permanência de veículos em sua unidade e, com isso, evitar custos com o pagamento dos novos valores de permanência. Para isso, foram utilizadas modernas ferramentas de Simulação em Computador para que as soluções mais adequadas pudessem ser implementadas com sucesso.

Palavras-chave: Logística interna; Lei do caminhoneiro; Simulação em computador; Eventos discretos.

\section{VEHICLE RESIDENCE TIME REDUCTION STUDY SUPPORTED BY COMPUTER SIMULATION}

\begin{abstract}
The steel industry goes through its worst period in Brazil and companies are looking for solutions to minimize costs and ensure operations with fewer resources. An important point in this search for potential gains, Logistics has meriting a special attention. This paper presents the efforts of AM Juiz de Fora, in partnership with Franco Arbeit Engineering, to reduce vehicle residence time in its site, avoiding the payment of the new permanence values. For this, modern Computer Simulation tools were used so that appropriate solutions could be successfully implemented.

Keywords: Internal logistics; Transport legislation; Computer simulation; Discrete events.

\footnotetext{
Doutor em Engenharia Mecânica e diretor da Franco Arbeit Engenharia, Campinas, SP, Brasil.

Gerente de Desenvolvimento de Processos Logísticos da ArcelorMittal, Belo Horizonte, MG, Brasil. Analista de Planejamento Logístico Senior da ArcelorMittal, Belo Horizonte, MG, Brasil.

Analista de Trefilação Senior da ArcelorMittal Juiz de Fora, Juiz de Fora, MG, Brasil.

Analista de Planejamento Logístico Senior da ArcelorMittal Juiz de Fora, Juiz de Fora, MG, Brasil.
} 
1 INTRODUÇÃO

Diante da pior crise da história da indústria brasileira do aço, os indicadores convergem para uma série de fatores conjunturais e estruturais que têm levado o setor a fechar diversas unidades desde o início de 2015. Nesse cenário, muitas empresas focam no ganho de eficiência e na redução de seus custos como estratégia para enfrentar turbulências. Se, por um lado, os empresários aguardam respostas do governo que apontem para uma saída, suas equipes técnicas seguem a trabalhar na busca de oportunidades que minimizem suas perdas. E, sem dúvidas, a Logística ainda é área fértil para se buscar essas oportunidades.

Conglomerado multinacional líder mundial na produção de aço, a ArcelorMittal emprega mais de 15.000 funcionários no Brasil, responsáveis por engrenar uma capacidade de mais de 11 milhões de toneladas por ano. Este trabalho foca na unidade Juiz de Fora, mas a ArcelorMittal conta com 29 outras no país para o beneficiamento de aço, distribuídas em São Paulo, Minas Gerais, Santa Catarina e Espírito Santo para oferecer produtos e soluções em aço nos segmentos longos e planos.

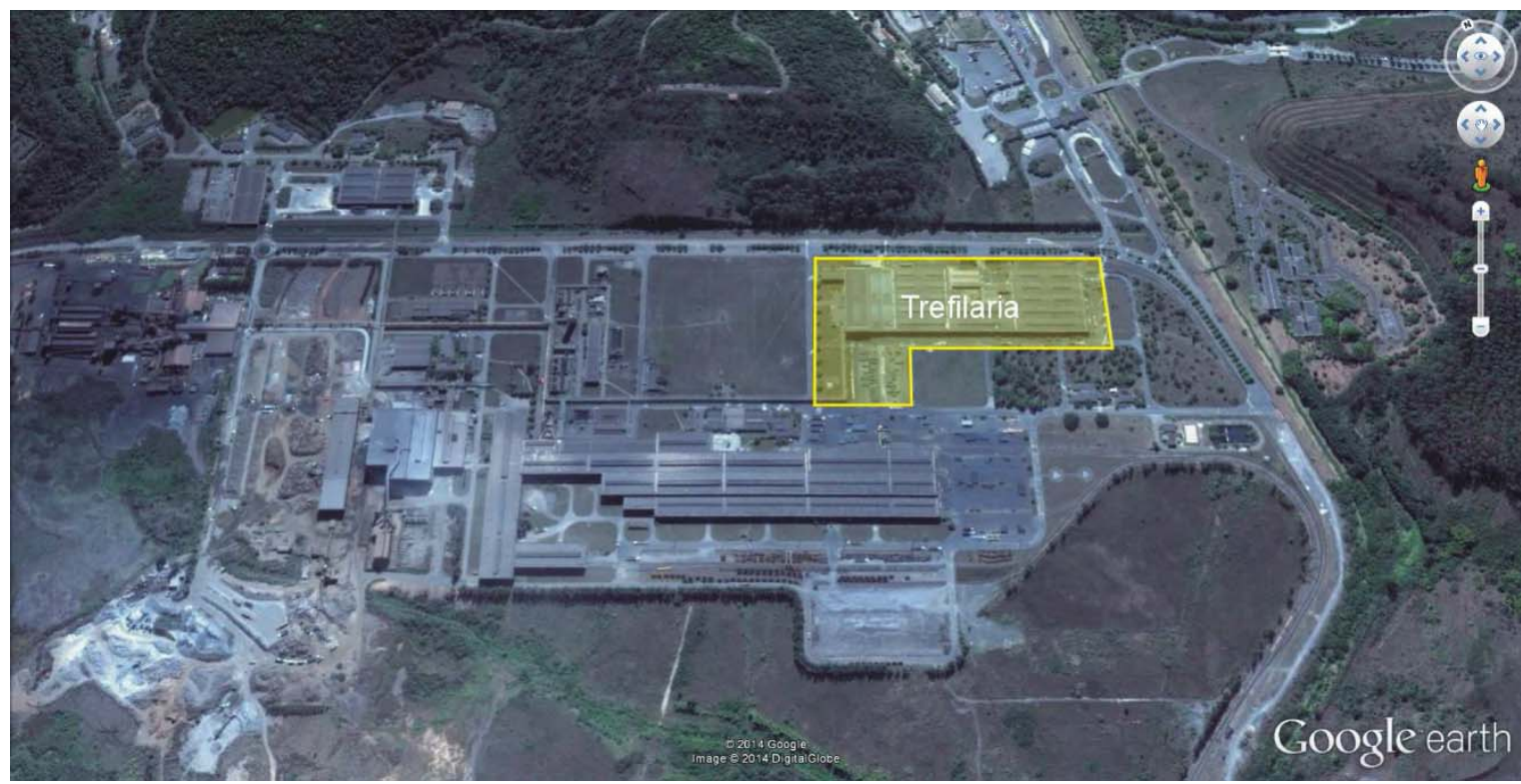

Figura 1 - Visão geral da Trefilaria da AM Juiz de Fora

A logística dessa usina conta hoje com uma estrutura montada para receber cerca de 87 caminhões por dia e expedir outros 107 veículos por dia. Além disso, há uma complexa movimentação interna, a qual é responsável pelo fluxo de 274 mil toneladas entre suas unidades.

Além de se adequar às condições econômicas do país, as empresas devem ficar de olho nas mudanças na área logística, uma vez que recentes medidas que regulamentam o setor de transporte aumentam ainda mais seus desafios. A Lei $n^{\circ}$ 13.103, publicada no dia 3 de março de 2015, já chamada de Lei dos Caminhoneiros, estabeleceu novas regras para o exercício da profissão.

Entre seus destaques estão as novas definições para o pagamento de estadia. De acordo com o parágrafo 5 , a Lei estabelece que:

"O prazo máximo para carga e descarga do Veículo de Transporte Rodoviário de Cargas será de 5 (cinco) horas, contadas da chegada do veículo ao 
endereço de destino, após o qual será devido ao Transportador Autônomo de Carga - TAC ou à ETC a importância equivalente a $R \$ 1,38$ (um real e trinta e oito centavos) por tonelada/hora ou fração. "

Com reajuste anual pelo INPC, o cálculo deve considerar a capacidade total do veículo, com pagamento retroativo à primeira hora. Além de contar com o prazo determinado de 5 horas para a operação, o embarcador e o destinatário da carga deverão fornecer ao transportador documento que comprove o horário de chegada do caminhão, o que demanda sistemas de agendamento de cargas e descargas, evitando-se o risco de passivos introduzidos pela Lei.

Fato é que algumas empresas ainda não estão preparadas para atender a nova Lei, tanto pela falta de estrutura para a rápida carga e descarga de veículos, quanto pela necessidade de sistemas para disponibilizar as informações necessárias. Análises preliminares apontam que uma usina do porte da AM Juiz de Fora poderá ter em seus custos logísticos aumentos na casa de dezenas de milhões de reais caso ações não sejam tomadas.

Assim, a ArcelorMittal focou nessas ações, sendo prioritário dentre seus projetos logísticos:

- A revisão e a otimização das operações de recebimento e expedição de suas unidades.

- A implantação dos sistemas de agendamento.

Este trabalho mostra especificamente os esforços da AM Juiz de Fora na redução do tempo de permanência de veículos de expedição em sua Trefilaria, gerando melhores condições operacionais e custos menores. Para isso, foi utilizada a experiência da Franco Arbeit Engenharia, empresa com dezenas projetos siderúrgicos realizados com o suporte da Simulação em Computador.

\section{MATERIAIS E MÉTODOS}

\subsection{Modelagem Conceitual}

Law \& McComas ${ }^{1}$ apontam a modelagem conceitual como uma importante ferramenta para apoiar o entendimento do problema em si e ajudar na validação de modelos de simulação. Assim, este trabalho partiu de um extenso trabalho de coleta de dados, tratamento estatístico e, sobretudo, modelagem conceitual.

Este projeto partiu de um extenso trabalho de mapeamento de fluxos, processos e operações, os quais foram representados por fluxogramas e Mapeamentos de Cadeia de Valor - VSM [Rother et al. ${ }^{2}$ ]. No total, foram estudadas as máquinas e seus diversos processos, áreas de armazenagem e, para garantir que a movimentação de materiais, pontes rolantes, pórticos e empilhadeiras operando em uma centena de diferentes fluxos.

Além de ajudar na especificação dos modelos computacionais, essa etapa de Modelagem Conceitual foi importante por auxiliar no melhor entendimento do sistema e na comunicação entre a operação e a equipe executora do projeto. Como exemplo dos modelos aqui desenvolvidos, a figura 2 mostra um dos processos analisados. 


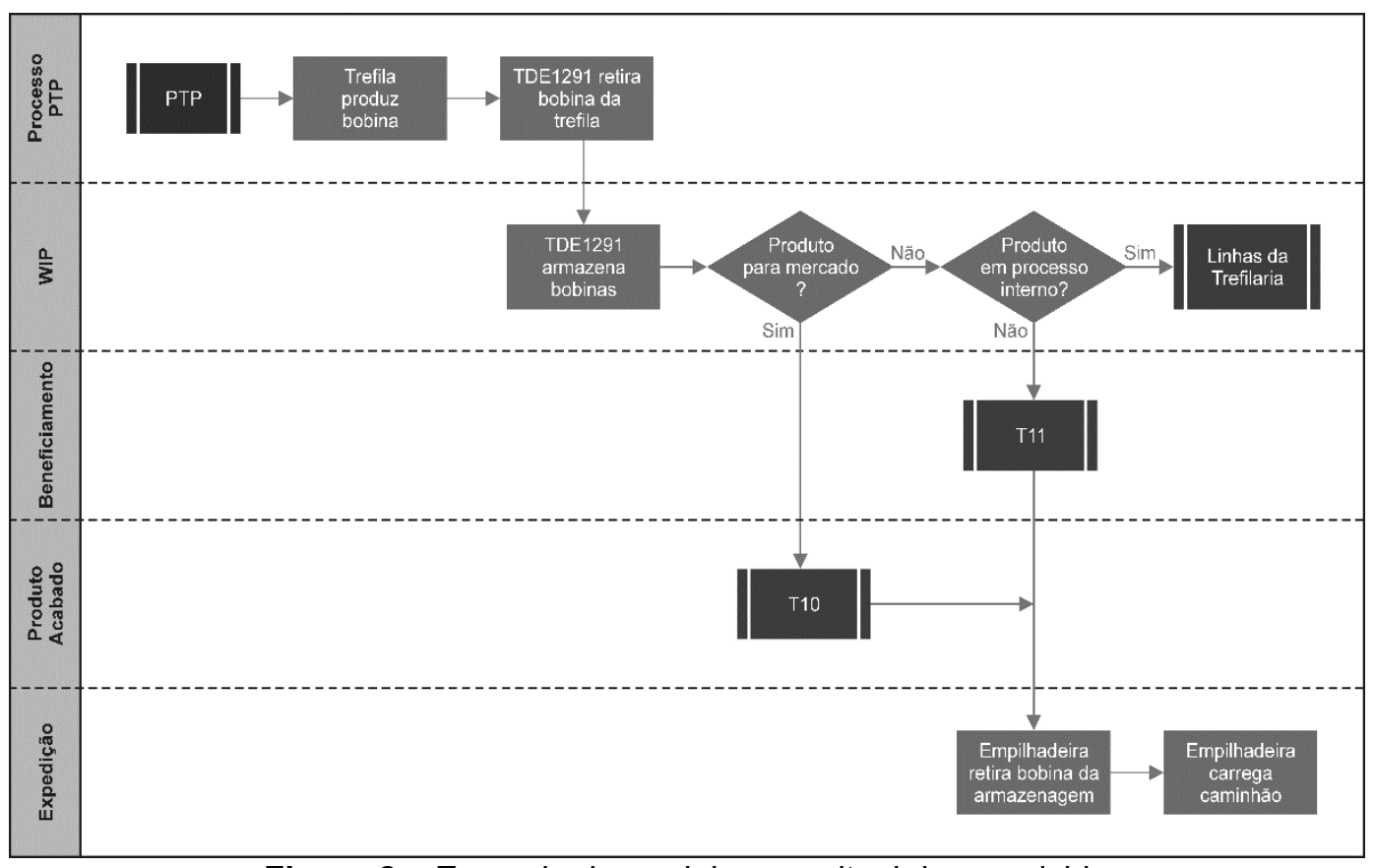

Figura 2 - Exemplo de modelo conceitual desenvolvido

\subsection{Simulação em Computador}

Enquanto metodologia que garante maior confiabilidade em projetos, simular significa submeter um modelo a repetidas observações [Banks ${ }^{3}$, sendo o modelo uma representação do mundo real com o objetivo de permitir a geração e análise de alternativas. Associada a Ciência da Administração, Pidd ${ }^{4}$ fala de uma definição onde um modelo tem o propósito de interpretar, mudar, gerir e controlar a realidade. Nesse sentido, a simulação pode ser descrita como uma ferramenta cujo objetivo é a consolidação de um melhor entendimento e/ou a identificação de melhorias num sistema.

Modelos de Simulação em Computador são comumente utilizados hoje em dia, sobretudo em projetos que envolvem recursos financeiros significativos e, para os quais, espera-se um retorno seguro sobre os valores investidos. Ainda em 1968, Anthavale ${ }^{5}$ já apontava a utilização da simulação como uma ferramenta para determinar o lead time de produtos e apoiar decisões de inventário e produção. Franco et al. ${ }^{6}$ apresentam as perspectivas para aplicação de ferramentas de simulação em indústrias siderúrgicas. Lima et al. $^{7}$ descrevem o modelo de Simulação em Computador desenvolvido em linguagem Java para o tratamento do complexo problema de manuseio e armazenagem de placas no pátio da ArcelorMittal Tubarão que alimenta seu Laminador de Tiras a Quente (LTQ). Rocha et al. ${ }^{8}$ descrevem um modelo computacional para tratar o fluxo de produtos laminados pelos entrepostos da UPV e propiciar o impacto de planos de expansão em seus equipamentos de movimentação.

O aplicativo selecionado para o desenvolvimento do modelo computacional aqui descrito foi o Arena, da Rockwell Automation, ferramenta de reconhecida robustez, a qual é líder mundial no mercado de Simulação em Computador e utiliza como base a linguagem de simulação SIMAN. Este modelo, cujo exemplo de animação pode ser visto na figura 3, considerou o fluxo de materiais da Trefilaria da AM Juiz de Fora, desde sua saída nas máquinas de trefila, passando por linhas como as de fabricação de recozidos, telas e treliças, indo até sua expedição rodoviária. 


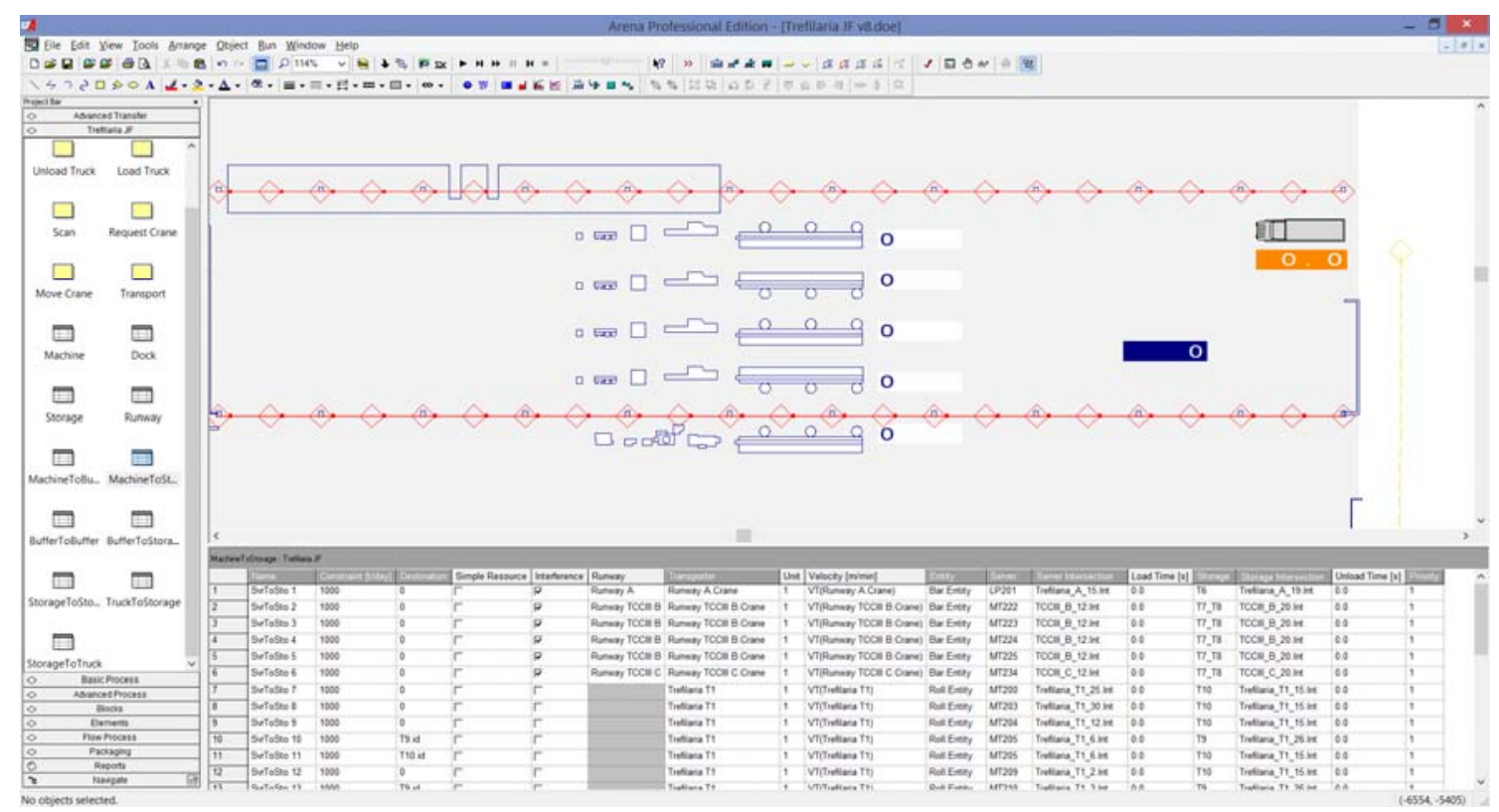

Figura 3 - Modelo de Simulação em Computador

Ao final da etapa de desenvolvimento do modelo computacional, chegou-se a um ambiente integrado com planilhas Excel capaz de analisar com agilidade configurações alternativas, submetendo-as a diferentes cenários.

\subsection{Análise de Riscos}

Prática comum nos projetos realizados nessa parceria da ArcelorMittal com a Franco Arbeit Engenharia, foi realizado um workshop para elaboração de uma Análise de Riscos. Ao buscar mudanças, uma empresa inova e aspectos de inovação aumentam ainda mais as incertezas. Isso leva a necessidade de ferramentas que aumentem a confiabilidade de que os resultados almejados serão alcançados.

Embora parte dessa garantia, no ponto de vista operacional, tenha sido obtida com a Simulação em Computador, aspectos mais abrangentes devem ser sempre considerados. Assim, foi aplicada uma metodologia baseada no Process Failure Modes and Effects Analysis - PFMEA [Rausand et al. ${ }^{9}$ ].

Poderosa ferramenta de priorização, utilizada para avaliar falhas específicas, o FMEA e sua variação para análise de processo são altamente eficazes para a identificação de atributos críticos, uma vez que quebram projetos complexos em etapas administráveis. Essa análise se caracteriza por contém duas sub análises, (1) a análise de modos de falha e efeitos e (2) a análise de criticidade.

\section{RESULTADOS E DISCUSSÃO}

Análises iniciais sobre a situação atual da logística internada da Trefilaria da AM Juiz Fora, apoiadas pelo mapeamento da modelagem conceitual e pelas simulações executadas, apontaram para necessidade de configurações alternativas. Ao se submeter o sistema atual a um cenário futuro de volumes, notou-se que os problemas já encontrados seriam aumentariam ainda mais a permanência dos veículos na usina e elevariam os custos logísticos.

De posse dessas necessidades, parâmetros de projeto foram definidos e transformados em alternativas para novas configurações físicas, bem como novos fluxos para o escoamento de produtos acabados, sua transferência entre os galpões 
e o carregamento para expedição. Essas alternativas consideraram tanto os aspectos operacionais quanto as definições estratégicas para as diferentes famílias de produtos.

Basicamente, foram simuladas uma dezena de configurações que envolveram a (1) pré-formação de cargas, (2) realocação de linhas de produção, (3) realocação de áreas de áreas de armazenagem e (4) construção de uma expedição centralizada.

Dentre as medidas de desempenho analisadas, umas das principais foi o tempo de ciclo de carregamento, como ilustrado na figura 4. Cada configuração alternativa foi então modelada e suas métricas foram comparadas para indicar as melhores soluções.

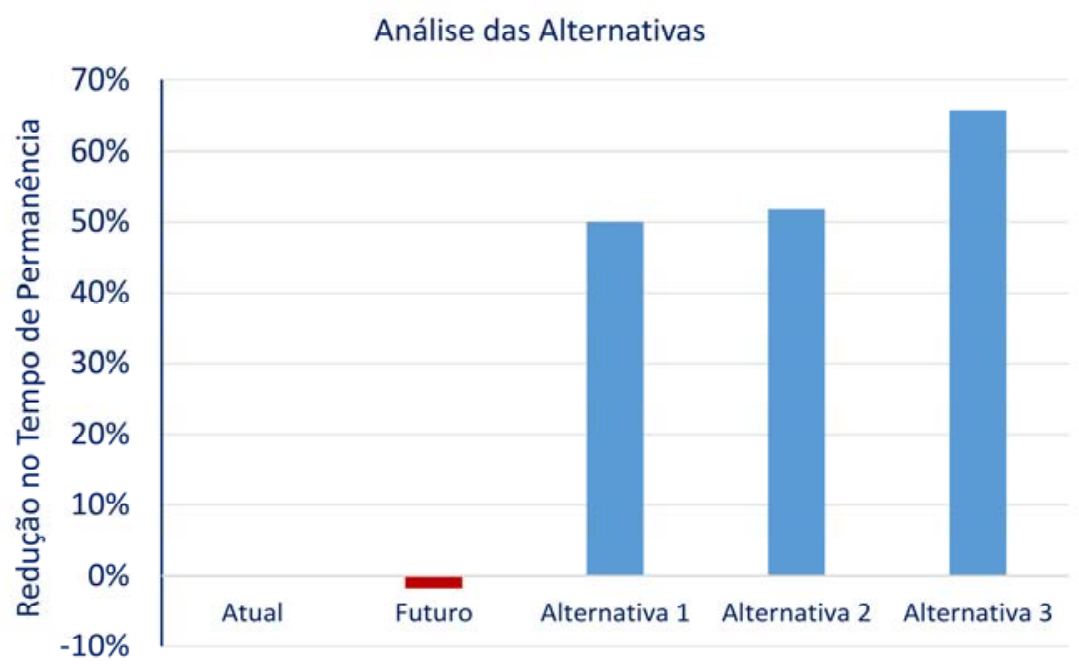

Figura 4 - Comparação do Tempo de Permanência das configurações alternativas

Conforme já era a expectativa, as simulações comprovaram que o tempo de ciclo dos veículos irá aumentar diante de cenários de volumes e mix futuros. Isso implicaria em maiores custos logísticos, justamente pelos valores agora definidos pela legislação apresentada anteriormente.

Os resultados simulados mostraram, ainda, que o investimento em algumas das configurações alternativas garante uma redução no tempo de permanência dos veículos na usina superior a $60 \%$. Em cenários estudados com baixos custos de implementação, essa redução chegou a $50 \%$.

Após ampla discussão dos resultados das alternativas futuras, e sua comparação com o retorno de investimento planejado para cada uma, foi apresentado um conjunto de processos, equipamentos e estruturas da Logística Interna, formando uma solução geral que minimizasse o tempo de ciclo dos veículos, otimizasse CAPEX e OPEX e garantisse uma melhor relação custo benefício. Dentre as soluções mais eficazes, a simulação demonstrou a importância de se realizar o pre picking para que cargas estejam preparadas na chegada dos veículos. Ainda, o modelo apontou para os benefícios de estruturas especialmente projetadas para minimizar o tempo de carregamento em si.

Esse conjunto foi então submetido à Análise de Risco, a qual apontou elementos que poderiam atrapalhar, ou até mesmo inviabilizar sua implementação. Para isso, foram realizadas uma série de reuniões técnicas onde operadores, analistas e gerentes discutiram diversos aspectos referentes a operacionalização das alternativas mais viáveis. 
Além dos riscos físicos da operação, alguns dos quais envolvem a segurança dos operadores e tiveram ações definidas como sendo de máxima prioridade, o Workshop de Análise de Riscos levantou e priorizou, segundo a metodologia adotada, alguns pontos de atenção, como:

- Adequação do sistema de agendamento à necessidade de formação de pré carga.

- Alguns equipamentos não possuem uma confiabilidade adequada para que o ganho almejado de produtividade seja alcançado (e.g. pontes rolantes sem inversor de frequência que aumentam muito o ciclo de suas operações).

- Necessidade de maior acuracidade em alguns armazéns.

\section{CONCLUSÃO}

As pressões econômicas aumentam diante da crise e operações mais enxutas se fazem necessárias. Os resultados do projeto aqui apresentado mostram que a Simulação em Computador continua sendo uma ferramenta importante cuja utilização tende a crescer em diversos setores, sobretudo os siderúrgicos, onde investimentos são elevados e seus retornos devem ser bem alinhados. Sendo assim, o modelo desenvolvido para representar a logística da Trefilaria da AM Juiz de Fora ajudou a encontrar melhores práticas para o alcance dos objetivos estratégicos da ArcelorMittal.

Destaca-se, ainda, que um fator fundamental para o sucesso deste projeto foi o trabalho integrado entre a equipe de projetistas da Franco Arbeit Engenharia e os colaboradores da ArcelorMittal maximizaram os resultados e minimizaram o tempo de resposta.

\section{Agradecimentos}

Aos colaboradores da ArcelorMittal e os responsáveis pelas áreas envolvidas, os quais não mediram esforços em fornecer detalhes de seu trabalho e subsidiar as análises do estudo.

A equipe da Franco Arbeit Engenharia, que efetivamente compreendeu os processos estudados e contribuiu na busca e validação das soluções.

\section{REFERÊNCIAS}

1 LAW, A. M., MCCOMAS, M. G. How to Build Valid and Credible Simulation Models. In: 2001 Winter Simulation Conference, Dec. 9 - 12, 2001, Arlington (VA). Proceedings of the 2001 Winter Simulation Conference, 2001.

2 ROTHER, M., SHOOK, J. Learning to See: Value Stream Mapping to Add Value and Eliminate Muda. Spi Edition. 102 pp. Cambridge (MA): Lean Enterprise Institute. 1999.

3 BANKS, M. C. What Is Modeling and Simulation? In: Principles of Modeling and Simulation: a multidisciplinary approach. p. 3-23. Edited by J.A. Sokolowski \& C.M. Banks. Hokoken, NJ: John Wiley \& Sons. 2009.

4 PIDD, M. Computer Simulation in Management Science. Fifth edition. 332 pp. West Sussex, England: John Wiley \& Sons. 2004.

5 ANTHAVALE, M. L. Manufacturing Lead Time Determination by GPSS Simulation. In: Second Conference on the Applications of Simulation, New York (NY), 2 - 4 Dec, 1968. FRANCO, G. N.; BATOCCHIO, A.; MORAES, G. C. Novas Perspectivas para a Simulação em Computador de Processos Siderúrgicos. In: $59^{\circ}$ Congresso Anual da ABM - Internacional, 2004, São Paulo (SP), 19 a 22 de julho de 2004. Anais do 59 
Congresso Anual da Associação Brasileira de Metalurgia e Materiais. São Paulo: Associação Brasileira de Metalurgia e Materiais, 2004. p. 1097-1105.

7 LIMA, J. S.; SIMÕES, J. A.; MOREIRA, A. C.; ZORZANELLI, L. R.; BARROS, R. S. F.; PERIN FILHO, C.; dos SANTOS, V.; FRANCO, G. N. Estratégia de Operação do Pátio de Placas do LTQ na ArcelorMittal Tubarão. In: 44 Seminário de Laminação Processos e Produtos Laminados e Revestidos, 16 a 19 de outubro de 2007, Campos do Jordão SP. Anais do 44 Seminário de Laminação - Processos e Produtos Laminados e Revestidos, 2007.

8 ROCHA, A. M., WHEHAIBE, V. E. K., SILVA, P. V. B., FRANCO, G. N. Análise das Restrições de Escoamento de Produtos Laminados na Usina Presidente Vargas. In $31^{\circ}$ Seminário de Logística da ABM, Belo Horizonte (MG), 19 a 22 de junho de 2012.

9 RAUSAND, M., HAYLAND, A. System Reliability Theory: Models, Statistical Methods, and Applications. Wiley Series in Probability and Statistics. 2nd Edition, 664 pp. Hoboken (NJ): Wiley-Interscience. 2004. 